\title{
Zwischen Sprachzerfall und Spracherhalt
}

\author{
Zum paradoxen Stimmengefüge in Texten von Tom Lubbock \\ und Wolfgang Herrndorf
}

Anna Katharina Neufeld

\begin{abstract}
"Sterben ist ein Trip. Als Desorganisation von Raum und Zeit, als Trennung von Körper und Identität, als Negation aller diskursiven und sozialen Regeln.» Thomas Macho, Todesmetaphern ${ }^{1}$
\end{abstract}

Am 16.11.2010 schrieb der Schriftsteller Wolfgang Herrndorf in seinem Blog Arbeit und Struktur:»Tom Lubbock stirbt und schreibt. «² Herrndorf bezieht sich auf das Memoir of living with a brain tumour ${ }^{3}$ des britischen Journalisten und Kunstkritikers Tom Lubbock, der, wie er selbst, an einem bösartigen Gehirntumor erkrankt war und seinen Sterbeprozess in tagebuchartiger Form für die Öffentlichkeit dokumentierte und kommentierte.

Lubbocks Memoir beginnt mit dem Satz: "This is about my dying and how my life got there. $\aleph^{4}$ Damit begreift Lubbock sein Leben bereits von seinem Ende her, indem er den Prozess benennt, um den es in seiner Denkschrift gehen wird: um sein Sterben. Dass er diesen Prozess mit der Zerstörung seines Sprachzentrums gleichsetzt, liegt nicht nur an der Lokalisierung des Tumors und seines raumgreifenden Wachstums, sondern auch daran, dass das Schreiben für ihn eine lebenswichtige Funktion hat. ${ }^{5}$ So schildert das Memoir, wie auch das

\footnotetext{
1 Thomas Macho, Todesmetaphern. Zur Logik der Grenzerfahrung, Frankfurt a.M. 1987, 408.

2 Wolfgang Herrndorf, Arbeit und Struktur, Berlin 2013, 158. Wolfgang Herrndorfs Blog Arbeit und Struktur (http://www.wolfgang-herrndorf.de) erscheint posthum 2013 als Buch im Rowohlt Verlag, Berlin. Die Seitenzahlen beziehen sich im Folgenden auf die Buchpublikation.

3 Der Artikel wurde am 7.11.2010 im Observer unter dem Titel Tom Lubbock: $A$ memoir of living with a brain tumour veröffentlicht und online gestellt, siehe: http:// www.theguardian.com/books/2010/nov/07/tom-lubbock-brain-tumour-language (30.5.2016).

4 http://www.theguardian.com/books/2010/nov/07/tom-lubbock-brain-tumourlanguage (30.5.2016).

5 Siehe: Tom Lubbock, Until Further Notice, I Am Alive, London 2012, 1 und http://www.theguardian.com/books/2010/nov/07/tom-lubbock-brain-tumour-
} 
posthum erschienene Buch Until Further Notice, I Am Alive (2012), die sehr detaillierten Auseinandersetzungen mit den verschiedenen Möglich- und Unmöglichkeiten des Sprechens, Lesens, Schreibens und Hörens. ${ }^{6}$

Die Überschneidungen zwischen Lubbocks und Herrndorfs Schicksal sind offensichtlich. ${ }^{7}$ Herrndorf scheinen Lubbocks »hybride Mischung aus Tagebucheinträgen und Notizen « ${ }^{8}$ ebenso zu bewegen wie auch seine Auseinandersetzung mit dem Verhältnis von Sterben und Sprache. ${ }^{9}$ Als Modell für Herrndorfs eigene Dokumentation kommen sie allerdings nicht in Frage. ${ }^{10}$ Herrndorfs Entscheidung, einen öffentlichen Blog zu führen, lässt Unterschiede erahnen. Das Blog ${ }^{11}$ suggeriert Formen der Lebendigkeit, die durch eine potentielle Unabgeschlossenheit vermittelt werden und der abgeschlossenen Verkörperungsform des Buches gegenüberstehen. ${ }^{12}$ Darüber hinaus verhandeln Blog und Buch Zeit ganz unterschiedlich: Die Inszenierung von Aktualität im Blog steht auch hier der abgeschlossenen Form des Buches entgegen, das immer schon ein Ende hat. ${ }^{13}$

\footnotetext{
language (30.5.2016).

6 Lubbock hielt während dieser Zeit sein Arbeitspensum als Kunstkritiker des Independent aufrecht und publiziert zwei Bücher: Great Works. 50 Paintings Explored, London 2011, und English Graphic, London 2012.

7 Des Weiteren lässt sich feststellen, dass auch die Kunst die beiden Autoren verbindet. Herrndorf war lange als Zeichner für das Satiremagazin Titanic tätig. Ähnlich wie Lubbock publizierte auch Herrndorf weiter nachdem er die Diagnose eines Gehirntumors erhalten hat: Sand, Berlin 2011, und, posthum erschienen, Bilder deiner großen Liebe, Berlin 2014.

8 Elke Siegel, "die mühsame Verschriftlichung meiner peinlichen Existenz«. Wolfgang Herrndorfs »Arbeit und Struktur» zwischen Tagebuch, Blog und Buch, in: Zeitschrift für Germanistik. Neue Folgen XXVI 2 (2016), 348-372, hier 350.

9 Herrndorf zitiert folgende Passagen: „My laguage works in ever decreasing circles. The whole of English richness is lost to me and I move fewer and fewer words around. I cannot count. At all. Marion and her embrace. Ground, river and sea. Eugene - his toys, his farm, his cars, his fishing game. Getting quiet. Names are going." Siehe: Arbeit und Struktur, 158f., und http://www.theguardian.com/books/2010/nov/07/ tom-lubbock-brain-tumour-language (30.5.2016).

${ }^{10}$ Siehe: Siegel, Herrndorfs "Arbeit und Struktur», 350.

${ }^{11}$ Herrndorf schreibt durchgehend von "das Blog«; im vorliegenden Aufsatz werde ich ebenfalls den sächlichen Artikel verwenden. Laut Duden kann auch die maskuline Form verwendet werden.

${ }^{12}$ Siehe: Natalie Binczek, Wo also ist der Ort des Textes? Rainald Goetz' Abfall für alle, in: Formen interaktiver Medienkunst. Geschichte, Tendenzen, Utopien, hg. v. Peter Gendolla/Norbert M. Schmitz/Irmela Schneider/Peter M. Spangenberg, Frankfurt a.M. 2001, 291-318, hier 296.

13 Siehe: Siegel, Herrndorfs »Arbeit und Struktur«, 363.
} 
Den Zerfall der Sprache beobachten und notieren beide Autoren auch Herrndorf stirbt und schreibt.Am 21.5.2013 notiert er in seinem Blog: »Dramatischer Sprachzerfall. [...] Mein häufigster Satz in Unterhaltungen: Was ist, was ich sagen will, nicht das, das andere Wort, das ohne mit dem, so was Ähnliches, das ja, nein, lateinische Wurzel, ja.$-{ }^{14}$ Wie also lässt sich Sprachzerfall dokumentieren, wenn die Sprache das Medium ist, in der geschrieben wird? Ich greife hier Herrndorfs Frage auf: Was wird gesagt, wenn Lubbock und Herrndorf von Sprachzerfall sprechen? Und wie schreiben sie darüber?

\section{Wer spricht?}

Lubbocks Memoir, erschienen im Observer (November 2010), folgt zwei Jahre später die Publikation Until further Notice, I Am Alive, die seinen Krankheitsverlauf in tagebuchartiger Weise noch ausführlicher dokumentiert. Dieser wird eine Introduction voran gestellt, die Lubbocks Frau Marion Coutts verfasst hat. Sie beginnt mit dem Satz: "Tom wrote this book. That in itself is astonishing. "15 $^{15}$ Dass Coutts Lubbock allerdings beim Schreiben hilft, thematisiert er im Verlauf seiner eigenen autobiografischen Notizen. ${ }^{16}$ Und auch Coutts betont in der Einleitung:

„Vast holes in language would suddenly appear, and great chunks of speech fall [sic] away. He strung words together like ropes across voids. I could always understand him, just. At a certain point I became his mouthpiece, although without being his brain I was a fraud. By October 2010, Tom could not write or read in any regular sense although he could comprehend text on a mysterious level $[\ldots]$. Our job was to help him say it. «17

Coutts macht hier deutlich, in welcher Funktion sie aktiv wird: als sein Sprachrohr ("mouthpiece»), also als ein Vehikel seiner Gedanken, welche sie durch Fragen, Wiederholungen, Frustration oder »inspired guesswork ${ }^{18} \mathrm{zu}$ begreifen versucht.

Die Frage, wer spricht, ist im Zusammenhang mit dem Erzählen vom Sterben von großer Bedeutung. Zeigt sich hier doch die Grenze der Selbstbestimmung, die sich in der Deutungshoheit einer

\footnotetext{
${ }^{14}$ Herrndorf, Arbeit und Struktur, 410-411.

${ }^{15}$ Lubbock, Until Further Notice, 1.

${ }^{16}$ Siehe ebd., 111, 123.

${ }^{17}$ Ebd., 2-3.

${ }^{18}$ Ebd., 3.
} 
Darstellung offenbart. So ermöglicht ein autobiografischer Schreibprozess die Thematisierung der Begegnung mit der Ohnmacht gegenüber der Krankheit, um darüber Autonomie zu erzielen, wie die Kulturwissenschaftlerin Corina Caduff in Bezug auf Darstellungen zum Sterben heute interpretiert. ${ }^{19}$ Coutts Bemühungen, Lubbocks Sprachrohr zu sein, um die Leerstellen (»voids«) zu überbrücken, lässt Lubbock auf der Textebene einen verständlichen Text produzieren.

Auch Herrndorf macht deutlich, dass Freunde ihm helfen: "Dramatischer Sprachzerfall. Unklar, ob die Worte schon schwinden oder ob nur Stress. Denn immer wieder gelingen fast fehlerfreie Sätze. [...] Jeden Satz im Blog mit größter Mühe zusammengeschraubt. Freunde korrigieren. $\aleph^{20}$ Neben den Korrekturen am Blog, die von mehreren Freunden begleitet werden, wird vor allem die Autorin Kathrin Passig wichtig. Sie wird zu seiner Nachlassverwalterin. Sie kommuniziert am 27.8.2013 per Twitter nicht nur seinen Suizid: "Wolfgang Herrndorf starb nicht am Krebs. Er hat sich gestern in den späten Abendstunden am Ufer des Hohenzollernkanals erschossen. $\mathbb{1}^{21}$ Passig führt außerdem, zusammen mit Herrndorfs Lektor Markus Gärtner, den Wunsch aus, dass aus dem Blog ein Buch wird.

Lubbock wie auch Herrndorf thematisieren demnach in ihren Texten, dass ihnen geholfen wird. Das ermöglicht ihnen weiter zu schreiben, trotz fortschreitenden Gehirntumors, der maßgeblich auf ihr Sprachzentrum Auswirkungen zeigt. Ausführungen und Beschreibungen ihrer epileptischen Anfälle oder dieVersuche, während eines solchen Anfalls Gedichte $\mathrm{zu}$ memorieren, stellen eine Form der Selbstbeobachtung dar, die beide in ihren Aufzeichnungen dokumentieren. Damit entsteht Distanz zu den realen Krankheitsprozessen. Caduff bezeichnet es als einen "Akt der Abtrennung", in welchem der Autor sein krankes Ich thematisiert, »indem er es von sich gleichsam abwirft und in den Text bannt ${ }^{22}$. Dies stellt sich in den beiden Texten als paradox dar: Ich und Sprache fallen hier zusammen. Das kranke Ich wird als Sprachzerfall in den Text verbannt, allerdings lässt sich dieser Sprachzerfall auf formaler Ebene nicht erkennen, vielmehr entstehen in beiden Fällen nachvollziehbare Texte.

\footnotetext{
${ }^{19}$ Corina Caduff, Szenen des Todes. Essays, Basel 2013, 152, 158.

${ }^{20}$ Herrndorf, Arbeit und Struktur, 411.

${ }^{21}$ https://twitter.com/kathrinpassig/status/372326405041713152 (30.5.2016). Im Blog wird der Schluss auf Grund von Überlastung erst später ergänzt. Dort heißt es: "Wolfgang Herrndorf hat sich am Montag, den 26. August 2013 gegen 23:15 Uhr am Ufer des Hohenzollernkanals erschossen." Siehe: http://www.wolfgang-herrndorf. de/page/2/ (30.5.2016).

22 Caduff, Szenen des Todes, 157.
} 
Die Frage, wer in den beiden Texten spricht, lässt sich also für beide Texte insofern beantworten, als dass ein paradoxes Stimmengefüge zum Vorschein kommt. Es kommt zu einem "Akt der Abtrennung" wie auch zu einer Hilfestellung für das schreibende Ich. Die Inanspruchnahme von Hilfe verhandelt dabei Sprache und ihren Zerfall in einem fein austarierten Prozess von Finden und Erfinden, Verstehen und Nicht-Verstehen. Gleichzeitig inszeniert sich das schreibende Ich über den "Akt der Abtrennung " auf der Textebene als selbstständig und autonom. So stellt sich neben der Frage, wer spricht, vor allem die Frage, welche Formen der Autonomie in den Texten verhandelt werden und welche Konstruktionen sie heranziehen?

\section{Formen der Autonomie}

\section{a. Psychohygiene und Code civil}

»Freundschaft war eines seiner großen Themen. Noch wichtiger aber war Wolfgang Herrndorf Autonomie, in der Kunst wie im eigenen Leben. Nun ist der großartige Schriftsteller gegangen - unbesiegt." Felicitas von Lovenberg ${ }^{23}$

Herrndorfs Aufzeichnungen lassen von Beginn an keinen Zweifel an seinem Entschluss, seinem Leben selber ein Ende setzen zu wollen: »Ich wollte nicht sterben, zu keinem Zeitpunkt [...]. Aber die Gewissheit, es selbst in der Hand zu haben, war von Anfang an notweniger Bestandteil meiner Psychohygiene. ${ }^{24}$ So lässt sich Herrndorfs Blog Arbeit und Struktur als programmatischer Umgang mit seinem Sterben als Form des "Autonomiegewinns ${ }^{25}$ lesen. Das Schreiben als Arbeit schafft nicht nur Struktur im Alltag, es ermöglicht ihm die Kommunikation mit der tödlichen Krankheit, welche eben »auch zur Krise der (Selbst-)Kommunikation wird « ${ }^{26}$.

Herrndorf betont mehrfach, sein Begriff von Leben bedeute die Fähigkeit zur Kommunikation..$^{27}$ Entsprechend ermöglicht das Blog $\mathrm{ihm}$, jene "Exitstrategie« zu formulieren, um zu wissen, dass - wie

\footnotetext{
${ }^{23}$ Felicitas von Lovenberg, Zum Tod Wolfgang Herrndorfs. Dieses Zuviel ist niemals genug, FAZ, 27.8.2013.

${ }^{24}$ Herrndorf, Arbeit und Struktur, 50.

${ }^{25}$ Siehe Caduff, Szenen des Todes, 152.

${ }^{26}$ Siegel, Herrndorfs "Arbeit und Struktur«, 349.

${ }^{27}$ Herrndorf, Arbeit und Struktur, 224, 335.
} 
er schreibt - »ich Herr im eigenen Haus bin $\aleph^{28}$. Seinen eindeutig gefassten Entschluss ergründet und führt Herrndorf im Verlauf seines Textes aus. Sei es, dass er sich mit dem Sterben anderer auseinandersetzt, beispielsweise mit Tom Lubbocks Memoir oder anderen literarischen und nicht-literarischen Vorbildern, ${ }^{29}$ sei es, dass er nach einem "praktikablen How-to ${ }^{30}$ recherchiert oder immer wieder die Schusswaffe thematisiert, mit der er letztlich seinen Suizid vollzieht. Autonomie und Schreiben stehen also in einem engen Zusammenhang. Die Thematisierung seines Sprachzerfalls ist dabei sowohl auf den Akt des Schreibens als auch auf den Akt des Suizids gerichtet und stellt für Herrndorf vor allem eine Frage der Zeitlichkeit dar. Ist es doch eine Frage der Zeit, wann er nicht mehr dazu in der Lage sein wird, die Dinge selbst in die Hand zu nehmen.

Mit der Buchpublikation von Arbeit und Struktur erfährt die Frage nach Zeitlichkeit eine weitere Dimension, die sich darin zeigt, dass Blog und Buch unterschiedlich mit der Frage von Zeit umgehen. Die Frage von Zeitlichkeit wird hier zum Moment der Grenzziehung zwischen Leben und Tod, die Herrndorf selber vornimmt und die sich im Umgang mit den beiden Medien, Blog und Buch, widerspiegelt. ${ }^{31}$ Mitte Juli 2013 inszeniert Herrndorf auf der Textebene seinen Tod. So spricht er am 16.7.2013 von sich und C. als "vergangen«: »Es gibt uns nicht mehr. Wir sind schon vergangen." Drei Tage später äußert er sich nicht nur über den Ort, an dem er gerne begraben sein möchte, er schreibt bereits von sich in der Vergangenheitsform:"[...] wenn es nicht vermessen ist, vielleicht ein kleines aus zwei T-Schienen stümperhaft zusammengeschweißtes Metallkreuz mit Blick aufs Wasser, dort, wo ich starb. ${ }^{32}$ Der Tod einer Libelle am 23.7.2013 lässt sich in diesem Zusammenhang wie seine eigene Beerdigung lesen, die er selber durchführt: „Sie ist tot. Ich schiebe den Leichnam in eine Streichholzschachtel. Mit C. bestatte ich die Libelle am Ufer. ${ }^{33}$ So verbannt Herrndorf nicht nur sein krankes Ich in den Text, er lässt dieses Ich, ganz im Sinne seiner

\footnotetext{
${ }^{28}$ Herrndorf, Arbeit und Struktur, 50, 79-81.

${ }^{29}$ Neben literarischen Texten, die im Text oft als Listen aufgeführt sind, setzte sich Herrndorf auch mit Filmen auseinander, wie beispielsweise Andreas Dresens Film Halt auf freier Strecke (D, 2011) oder das YouTube-Video der einstündigen Dokumentation Choosing to Die, in der sich der Schriftsteller Terry Pratchett mit der Möglichkeit der aktiven Sterbehilfe beschäftigt. Siehe: Herrndorf, Arbeit und Struktur, 44, 281, $208,314,408$.

${ }^{30}$ Herrndorf, Arbeit und Struktur, 50.

${ }^{31}$ Siehe Siegel, Herrndorfs "Arbeit und Struktur«, $363 f$.

${ }^{32}$ Siehe Herrndorf, Arbeit und Struktur, 421.

${ }^{33}$ Ebd., 423.
} 
Vorstellung eines Endes, im Text sterben, ohne allerdings die Agonie des Sterbeprozesses zu schildern.Vielmehr wird über den Gebrauch des Präteritums der Tod des Text-Ichs erzählt. Sein eigener realer Tod wird mit der Überschrift Schluss verdeutlicht und in dem Textabschnitt nur Ort, Zeit und Todesart mitgeteilt.

Die Funktion des Blogs besteht allerdings nicht allein darin, dass Herrndorf die Zeit selber in die Hand nimmt und so sein Text-Ich sterben lassen und begraben kann. Das Blog verweist auf einen weiteren Aspekt, der vor allem für die darauffolgende Buchpublikation wichtig ist. Das Blog erfüllt eine soziale Funktion. ${ }^{34}$ Es öffentlich zu machen, gründet auf einem Zusammenspiel verschiedener Einflüsse, wie Herrndorf im Blog selbst reflektiert. War das Blog anfangs nur seinem Freundeskreis zugänglich, wurde es bald auf Drängen von Freunden ${ }^{35}$ und zeitgleich mit der Veröffentlichung seines Romans Tschick im September 2010, unter dem Titel Arbeit und Struktur, zu einem öffentlich geführten digitalen Tagebuch. ${ }^{36}$ Seinen Erfolg als Autor von Tschick reflektiert er hier: »Ich bin Schriftsteller«, wie er in seiner Rückblende, Teil 1: Das Krankenhaus schreibt. ${ }^{37}$ Sein Erfolg schafft nicht nur eine ökonomische Unabhängigkeit, es katapultiert ihn auch aus seinem winexistenten Sozialleben" in ein öffentliches Leben als gefragter Autor. Damit betritt er einen öffentlichen Raum, der es ihm ermöglicht, anhand seiner eigenen Geschichte seine persönlichen Vorstellungen von Sterbehilfe zu formulieren. Wie bereits erwähnt, war die kurz nach seinem Suizid erschienene Buchpublikation des Blogs bereits zu seinen Lebzeiten als Wunsch von ihm formuliert worden. "Bei Bedarf « und wenn er seine "Aufzeichnungen nicht selbst bis zuletzt durchhalte ${ }^{38}$, solle es, neben dem kritischen Lektorat, ein Nachwort zum Buch geben. Laut Gaertner/Passig hatte Herrndorf Vorgaben anlehnend an das Stendhal Zitat - "Ich wollte, dass dieses Buch wie der Code civil geschrieben sei. In diesem Sinne sind alle dunklen oder unkorrekten Sätze zu korrigieren. $\aleph^{39}$ - formuliert. Gärtner und Passig verdeutlichen, dass es wenig zu korrigieren gab. Der

\footnotetext{
${ }^{34}$ Siehe: Siegel, Herrndorfs "Arbeit und Struktur«, 57. Das Blog, so die Germanistin Elke Siegel, hat insofern eine soziale Funktion, dass das Schreiben als Arbeit, strukturgebenden ist, was allerdings, wie Herrndorf mehrfach betont, seine "Asozialität" verstärkt. Gleichzeitig öffnet sich Herrndorf über die Arbeit am Blog in das Soziale. Siehe: Arbeit und Struktur, 156, 91, 125, 283.

${ }^{35}$ Als Freunde spielen eine entscheidende Rolle die Internetaktivisten Sascha und Meike Lobo, Holm Friebe und Katrin Passig.

36 Siehe Arbeit und Struktur, 443 und Siegel, Herrndorfs "Arbeit und Struktur«, 348.

${ }^{37}$ Herrndorf, Arbeit und Struktur, 104.

${ }^{38}$ Ebd., 444.

${ }^{39}$ Ebd.
} 
Zusammenhang zwischen Code civil und Herrndorfs Vorgaben wird hier nicht weiter ausgeführt. Die Erwähnung des Code civil allerdings, als Meilenstein der bürgerlichen Gesetzgebung, lässt sich verknüpfen mit Herrndorfs Wunsch im Nachwort, eine medizinisch-fachliche Beschreibung seines Todes abgedruckt zu sehen. ${ }^{40}$ Damit übernimmt das Buch die Funktion, im Nachleben zum Blog zu einem "Mittel zur Selbsthilfe " ${ }^{41}$, also zu einer Anleitung zum Suizid mit Schusswaffe, zu werden, die medizinisch-fachlich die Position der Waffe beschreibt.

Stellt das Schreiben im Netz, wie der Germanist Uwe Wirth behauptet, eine Konvertierung eines »in real life gegebenen Körpers in virtuelle, digitalisierte Netzkörper «2 dar, so hat Herrndorf nicht nur im Blog seinen Freitod mit seinen eigenen Worten inszeniert, er hat darüber hinaus mit der Buchpublikation ein Erbe geschaffen. »Der 'Lebendigkeit des online-publishing wird die Buchpublikation als >Totenform` gegenübergestellt « ${ }^{43}$, so Wirth. In diesem Sinne gleicht das Buch einem Manifest, was auch daran liegt, dass Herrndorf sich auf diese Weise implizit in die aktuellen politisch geführten Debatten um Sterbehilfe einmischt. Gleichzeitig ermöglicht es die Wiedergeburt des verstorbenen Blog-Autors als Rowohlt-Autor. ${ }^{44}$ Dass es gerade Herrndorfs Schusswaffe ist - und keine weiteren Objekte, seien es Manuskripte, Skizzen oder Notizen - , die sich seit April 2016 als Objekt im Tresorschrank des Literaturarchivs in Marbach befindet, ${ }^{45}$ verdeutlicht einmal mehr, dass der Suizid sein Überleben als Autor auf mehreren Ebenen gesichert hat.

\footnotetext{
40 Ebd., 445.

${ }^{41}$ Katrin Passig, Mein Wille geschehe, in: Süddeutsche Zeitung Magazin, Heft 49/2013.

${ }^{42}$ Uwe Wirth, Neue Medien im Buch, in: Chiffre 2000 - Neue Paradigmen der Gegenwartsliteratur, hg. v. Corina Caduff/Ulrike Vedder, München 2005, 171-184, hier 172.

43 Ebd., 182.

${ }^{44}$ Siehe ebd., 184.

${ }^{45}$ Siehe: Julia Encke, Herrndorfs Revolver, in: Frankfurter Allgemeine Sonntagszeitung, 10. April 2016, Nr. 14, 41.
} 


\section{b. Der Schwindel als dritte Instanz in Lubbocks Memoir}

"Life is walking tiptoe over land mines.

We never know what's coming [...]"

Siri Hustvedt, The Blazing World ${ }^{46}$

Die Inszenierung eines selbstbestimmten Text-Ichs ist in beiden Texten ein Paradox, da es in beiden Texten an den geschilderten Sprachzerfall gekoppelt ist. Nur durch eine dritte Instanz ist das Schreiben bei gleichzeitigem Sprachzerfall möglich, wie Lubbock und Coutts oder auch Herrndorf und Gärtner/Passig nie verheimlichen. Die Funktion dieser dritten Instanz ist dabei nicht nur abhängig von ihrer Inszenierung, sondern auch, wie bei Herrndorf deutlich wird, von der Wahl des Mediums und der Todesart.

Die Funktion der dritten Instanz in Lubbocks Texten ist ein Dazwischen, oder wie ich anlehnend an Coutts verdeutlichen möchte, ein Schwindel. Den Begriff "fraud «/Schwindlerin, den Coutts in Bezug auf ihre Person benutzt, stellt ihre Bemühungen dar, ihre Mittelbarkeit als Sprachrohr hinsichtlich Lubbocks Gedanken (»brain«) sichtbar zu machen. Gleichzeitig wird kurz vorher erwähnt, wie Coutts den Vorgang des Helfens erlebt: als »vertiginous / schwindelerregend ${ }^{47}$.

Der Schwindel ist hier also doppeldeutig und durchaus positiv $\mathrm{zu}$ verstehen. Er besteht einerseits darin, dass - im übertragenen Sinn - Lubbock/Coutts die Bodenhaftung einer erlernten und automatisierten Sprache, des Sprechens, Schreibens und Hörens immer wieder verlieren, sie zu taumeln glauben, das Gleichgewicht verlieren. Lubbock führt in seinem Text immer wieder die Suche nach dem Verhältnis von Denken, Verstehen und Sprache aus, das sich in seinem Sterbeprozess so immanent verändert: Der wiederkehrende Verlust von Alltagssprache, die in einer automatisierten Form verfährt, ${ }^{48}$ das konzentrierte Suchen nach Worten, wenn sich Sprachpannen ${ }^{49}$ einstellen, oder die Feststellung, dass die Kohärenz eines Plots, die Chronologie einer Erzählung nicht mehr nachvoll-

\footnotetext{
${ }^{46}$ Siri Hustvedt, The Blazing World, New York 2014, 13.

${ }^{47}$ Marion Coutts beschreibt in der Einleitung, wie schwindelerregend die Auseinandersetzung mit Tom Lubbocks Sprache war. Coutts versuchte, Muster zu erkennen, nach denen sie sich richten konnten, die aber innerhalb von Tagen sich wieder veränderten und erneut entdeckt und aufgedeckt werden mussten. Siehe Lubbock, Until Further Notice, 2.

${ }^{48}$ Ebd., 22.

${ }^{49}$ Als Sprachpannen beschreibt Lubbock beispielsweise die Unmöglichkeit, Worte richtig aussprechen zu können, da ihm die Buchstaben durcheinander geraten. Siehe ebd., 81.
} 
zogen werden kann, es aber trotzdem eine "mysteriöse " Form des Verstehens gibt. ${ }^{50}$

Andererseits kann der Schwindel als eine Form von Wahrheit gelesen werden, die einer anderen Logik als der normativen folgt. Lubbock schreibt einige Monate, nachdem er die Diagnose erhalten hat:

"There is a narrative problem. I don't know at what stage, and in what story, I am. [...] Some conditions such as mine would be clearly moribund, and some would be looking for recovery, and some would be looking for reasonable life prolongation, but I don't know what my narrative frame is - well, moribund, surely enough, but span is all. $\aleph^{51}$

Lubbock spielt hier auf die Fortschritte im medizinischen Bereich an, welche das Eingreifen in Sterbeprozesse ermöglichen. Dies bedeutet gleichzeitig, dass Sterbeprozesse in vielen Fällen gestaltbar und abhängig von Entscheidungen geworden sind und hinsichtlich des Umgangs mit diesen Möglichkeiten abgewogen werden müssen. Dass dabei allerdings der Ausgang jedweder Entscheidung trotzdem ungewiss bleibt, verdeutlich Lubbock, wenn er von einem "narrative problem " spricht oder erkennt: »I don't know what my narrative frame is." Es gibt nicht die eine Erzählung zum eigenen Sterben, es gibt mehr als diese eine. Die Möglichkeiten zur Gestaltung des Sterbeprozesses eröffnen mehr als eine Option des Erzählens. Dabei gilt es, diese verschiedenen Erzählungen im Blick zu behalten ${ }^{52}$ und in ihrer Gleichzeitigkeit auch auszuhalten. Die "narratives" werden allerdings dem Tod voraus gedacht: Es wird eine Zukunft imaginiert (»imagining futures «) ${ }^{53}$, die nur als Fiktion bestehen kann und so ihre eigene Wahrheit trägt. Das heißt, der Schwindel, der hier für die Möglichkeit, überhaupt einen Text herstellen zu können, charakteristisch ist, besteht aus den vielen fiktiven "narratives", die Lubbock mit Hilfe seiner Frau während seines Sterbeprozesses entfaltet. »His task was, in his own words", wie Coutts posthum betont, "a lesson in imagination, in self-imagination. $\aleph^{54}$

Lubbock erschreibt sich also einen paradoxen Raum, dessen Inhalt den Zerfall seines Sprachvermögens wie auch den Zusammenhalt dieses Zerfalls thematisiert. Er bezeichnet diesen Prozess als Aben-

${ }^{50}$ Siehe ebd., 3, 143.

51 Ebd., 61-62.

52 „I need to hold several perspectives together.« Ebd., 40.

${ }^{53}$ Ebd., 37.

${ }^{54}$ Ebd., 1. 
teuer sowie als Unglück. ${ }^{55}$ Zerfall und Zusammenhalt gehören in einer reziproken Dynamik zusammen und bleiben zugleich variabel, vielfältig und unvorhersehbar. ${ }^{56}$ Diesen Raum bezeichnet Lubbock als "halfway house of life und charakterisiert ihn als "inherently transitional " / in sich vorübergehend $d^{57}$. Er greift eben jene verschiedenen Perspektiven und Fiktionen auf, die Lubbock hinsichtlich seines Sterbeprozesses imaginiert. Das "halfway house of life" kann als Zwischenraum bezeichnet werden,$^{58}$ welcher durch die gegenwärtige Debatte ums Sterben - die kontrovers diskutierten ethisch-moralischen Fragen zur Sterbebegleitung und -hilfe im Umgang mit der "Janusköpfigkeit" der Intensivmedizin ${ }^{59}$ - allerdings erst entstanden ist. Die Frage der Autonomie des Sterbenden besteht hier also in Bezug auf die Haltung ("attitude"), die Lubbock gegenüber den verschiedenen Perspektiven einnimmt, die er als Imaginationen entwirft.

"At the moment I feel torn between pictures: the role of the surviving, and the role of the dying. Both have a self. I can hope to extend my life for a little longer, and I can recognise that my ending is in prospect. They have the same length, of course. Only their attitudes alter. ${ }^{60}$

Dabei stellt Lubbock fest, dass jener Zwischenraum eine elementare Weisheit (»elementary wisdom») in sich trägt: »This halfway house is a common state of life, which any of us may find ourselves in, and therefore must find ways of living in. ${ }^{61}$ Offen bleibt an dieser Stelle, ob sich Lubbock in Bezug auf die Weisheit hier auf das Wesensmerkmal des Menschen bezieht, dass wir alle Sterbliche sind. Eine Erkenntnis, die er an verschiedenen anderen Stellen deutlicher thematisiert. ${ }^{62}$ Oder, ob er das "halfway house of life» als Nachden-

\footnotetext{
${ }^{55}$ Ebd., 5, 139. lubbock-brain-tumour-language (30.5.2016).

${ }^{57}$ Lubbock, Until Further Notice, 19. zin, München 2010, 293.

${ }^{60}$ Lubbock, Until Further Notice, 129.

${ }^{61}$ Ebd., 19.

${ }^{62}$ Siehe ebd., 12, 57, 62, 73, 85, 87, 142.
}

${ }^{56}$ Siehe ebd., 19, 75, und http://www.theguardian.com/books/2010/nov/07/tom-

${ }^{58}$ Siehe Anna Katharina Neufeld, Der Sterberaum als Bühne des Übergangs. RaumInszenierungen in palliativmedizinischen und autobiografischen Texten, in: Zeitschrift für Germanistik. Neue Folge XXV, 3 (2015), 514-524, hier 520.

${ }^{59}$ Der Palliativmediziner Michael de Ridder weist auf die »janusköpfige Medizin" hin, die durch ihre Fortschritte das wirksame Eingreifen in Sterbeprozesse erst ermöglicht hat und damit den Menschen vor die schwierige Situation stellt, über sein Sterben entscheiden zu müssen. Siehe Michael de Ridder, Wie wollen wir sterben? Ein ärztliches Plädoyer für eine neue Sterbekultur in Zeiten der Hochleistungsmedi- 
ken über seinen Schreibprozess betrachtet, der allerdings in seinem Memoir und Until Further Notice, I Am Alive im Zusammenhang mit seinem Sprachzerfall steht, also seinem Sterbeprozess selbst. Beide Bezüge lassen sich kaum voneinander getrennt denken. Vielmehr bleiben auch sie als mögliche Perspektiven stehen und verdeutlichen umso mehr, wie über die reziproken Dynamiken zwischen den Fiktionen des Entstehens und Zerfallens, Lubbock immer wieder neu vor die herausfordernde Frage gestellt wird, wie er sich in seiner eigenen Geschichte positioniert.

\section{Ausblick}

"That his dying would be so exactly continuous with his living, one and the same, was not something I understood in advance. I had thought that death was a separate, foreign state. It is, but it follows the contours of our own terrain." Marion Coutts in Until Further Notice, I Am Alive ${ }^{63}$

Tom Lubbock und Wolfgang Herrndorf verlagern auf paradoxe Weise ihr krankes Ich in einen Text, um sich mit ihrem Sterbeprozess auseinanderzusetzen. Dabei generieren sie auf unterschiedliche Art ihre Form der Autonomie, die nicht zuletzt daraus resultiert, welche Positionen sie hinsichtlich ihres Sterbens einnehmen und welches Medium der Veröffentlichung sie wählen. Das heißt die Darstellungsform, das Wie-stellen-sie-ihren-Sterbeprozess-dar, drückt den Weg zur eigenen Haltung aus. Lubbocks und Herrndorfs Autonomie manifestiert sich also in einem Übergang, der sich als Zwischenraum offenbart und den Lubbock "halfway house of life" nennt und Herrndorf in der Auseinandersetzung mit seiner sogenannten "Exitstrategie" ausformuliert. Dieser Zwischenraum ist ein Bereich des Möglichen, zu dem beide Autoren letztlich divergierende Ansichten vertreten. Herrndorf wählt den zweifachen Suizid und überlebt gleichsam in der "Totenform" des Buches und als Autor eines Manifests mit medizinisch-fachlicher Anleitung für den Suizid mittels Schusswaffe. Lubbock hält die Imaginationen seines Lebensendes zusammen und beendet seine Aufzeichnungen mit den Sätzen: »The final thing. The illiterate. The dumb. Speech? Quiet but still something? Noises? Nothing? My body. My tree. After that it becomes simply the world. ${ }^{64}$ Er überlebt in den Worten seines

\footnotetext{
${ }_{63}$ Ebd., 9.

${ }^{64}$ Ebd., 144-145.
} 
Buchtitels Until Further Notice, I Am Alive, die seinem realen Tod widersprechen.

Die ausführlichen Schilderungen ihres Sprachzerfalls behandeln also das filigrane Gleichgewicht von Abhängigkeit und Autonomie, das gerade für sie als Schriftsteller mit einem Gehirntumor in der Auseinandersetzung mit dem Zerfall von Sprache und Schreiben virulent wird. So stellt sich für beide, Lubbock und Herrndorf, Autonomie immer im Verhältnis von Hilfestellungen und ihrer Inanspruchnahme dar. Als ein schwindelerregendes Wechselspiel von der Suche nach Worten, dem Memorieren von Sätzen, dem Sichtbarmachen von Automatismen, dem Hinterfragen von Sprachkonventionen, drückt es zugleich eine Reflexion auf den eigenen Schreibprozess aus. So zeigen die beiden posthum erschienenen Publikationen "die Totenmaske der Konzeption ${ }^{65}$ auf, was Walter Benjamin als dreizehnte These seiner Techniken des Schriftstellers bezeichnet. Die Germanistin Elke Siegel weist in dem Zusammenhang auf die griechische Bedeutung von Maske, persona, hin, »durch die das Persönliche spricht ${ }^{66}$. Dass der Tote und auch die Maske ambivalente Momente von Anwesenheit und Abwesenheit entstehen lassen, haben der Kulturwissenschaftler Thomas Macho mit seinem "Leichenparadox" und der Kunsthistoriker Hans Belting mit seiner Bild-Anthropologie bereits mehrfach betont. Macho spricht von dem "Rätsel, einer unerklärliche[n] Verdopplung ", das mit dem Betrachten eines toten Körpers einhergeht: Scheint dieser zwar identisch mit dem Verstorbenen zu sein, ist er aber ebenso offensichtlich auch nicht mehr identisch mit ihm. ${ }^{67}$ Die Maske greift eben diese Ambivalenz durch ihre Form von Verhüllung und Enthüllung auf, wie Belting verdeutlicht. ${ }^{68}$ So ist es auch jenes paradoxe Stimmengefüge, das aus den Texten Arbeit und Struktur sowie Until Further Notice, I Am Alive zu uns posthum spricht. Dies geschieht weniger in der Form einer mahnenden ars moriendi als vielmehr in Form eines Vexier-Bildes der letzten Worte, dessen elementare Weisheit für das Leben wie auch das Sterben gilt.

- Anna Katharina Neufeld promoviert im Bereich Kulturwissenschaft und Germanistik an der Humboldt-Universität zu Berlin zum Thema Sterben erzählen. Zum Zusammenhang von zeitgenössischen Sterbenarrativen und einer neuen Sterbekultur. Sie ist Stipendiatin des Evangelischen Studienwerks e.V.

\footnotetext{
${ }_{65}$ Walter Benjamin, Einbahnstraße, Berlin 1928, 33.

${ }^{66}$ Siegel, Herrndorfs »Arbeit und Struktur«, 66.

${ }^{67}$ Siehe Macho, Todesmetaphern, 195ff., und Thomas Macho, Wir erfahren Tote, keinen Tod, in: Der Tod. Eine Lesebuch von den letzten Dingen, hg. v. Rainer Beck, München 1995, 293-298, hier 295.

${ }^{68}$ Siehe: Hans Belting, Bild-Anthroplogie. Entwürfe für eine Bildwissenschaft, München 2001,153f.
} 